\title{
Fibre-optic sensors in biomedical sciences
}

\author{
Otto S. Wolfbeis
}

Analytical Division, Institute of Organic Chemistry, Karl Franzens University, 8010 Graz, Austria

\begin{abstract}
Optical fibres can be used for remote sensing of chemical and physical parameters. The usual approach is to provide the fibre end with a suitable indicator chemistry or a material that responds to the parameter of interest. In contact with the sample, the intensity of reflected, scattered, or re-emitted light is the analytical signal. Aside from several advantages of optical sensors over electrochemical ones (for instance, lack of reference cells and immunity towards electromagnetic interferences), the use of fibres can be advantageous when the samples are inaccessible, for instance, in case of in-vivo experiments.

The principles of waveguide spectroscopy are briefly outlined, and representative examples of fibre-optic sensors for temperature, oxygen, pH, carbon dioxide and electrolytes are given. Apart from these parameters, which also may be sensed by other techniques, optical sensors have been developed that are based on quite new principles and which can make them useful for sensing glucose, enzyme activities, anaesthetics and antigenantibody interactions. Potential fields of application are presented, and current trends and concepts discussed in a final section.
\end{abstract}

\section{INTRODUCTION}

Continuous sensing of analytes is a matter of growing interest by virtue of the realtime nature of most sensors. A sensor is a device that is able to indicate continuously and reversibly the concentration of an analyte or a physical parameter. Various sensing principles compete successfully with each other (Table 1). Among the electrochemical methods, potentiometry is probably most widely used at present. The best-known example is the glass electrode which has been used successfully over decades. More recently, $\mathrm{pH}$ electrodes have been applied as transducers for bioprocesses in which protons are produced or consumed.

Optical methods offer an interesting alternative to electrochemical sensors. Thus, absorptiometry, fluorimetry, infrared, and Raman spectroscopy have already been used for long periods and are more or less established in the analytical laboratory. But despite the manifold available methods there has been one limitation so far. The object of interest is occasionally not available in a form that fits the sample compartment of the instrument. In process control or in nuclear power plants, on the other hand, it is desirable to perform analysis in the benign environment of a laboratory rather than at places that are difficult to reach and where operators as well as expensive instrumentation may be exposed to hazardous conditions.

The remedy for this situation is the fibre-optic waveguide, which allows the transportation of an optical signal over large distances from the sample to the meter $(1,2)$. Fibres are robust and can therefore be exposed to varying, even hostile conditions, whereas the rather sensitive optical instrument can remain in a central laboratory. Plastic fibres are biocompatible so that they can be applied to invasive methods in physiology studies and medicine.

Fibres are an outgrowth of the communication industry (2). Suggested as a means for longdistance communications in 1968, they are now gradually replacing classical electric cables because they are cheaper than copper wires and now have signal attenuations as low as less than $1 \mathrm{~dB}$ per kilometre at 1.5 micrometre wavelength. A first transatlantic cabsu is under construction. 
Table 1. Sensor and Transducer Types Other than Optical Ones Exploited in Analytical and Clinical Chemistry

\begin{tabular}{|c|c|c|c|}
\hline Sensor Type & Principle & $\begin{array}{l}\text { Typical Sensing } \\
\text { Application }\end{array}$ & $\begin{array}{l}\text { Typical Tranducer } \\
\text { Application }\end{array}$ \\
\hline Glass electrode & potentiometry & $\mathrm{pH}$ & $\begin{array}{l}\mathrm{CO}_{2}, \mathrm{NH}_{3} \text {, enzyme } \\
\text { electrodes, titrimetry } \\
\text { (pH stat methods) }\end{array}$ \\
\hline Pt electrode & potentiometry & redox status & - \\
\hline $\begin{array}{l}\text { Field-effect } \\
\text { transistors }\end{array}$ & potentiometry & Ions and gases & $\begin{array}{l}\text { enzymes, substrates, } \\
\text { antibiotics, immuno- } \\
\text { systems }\end{array}$ \\
\hline $\begin{array}{l}\text { Ion-selective } \\
\text { electrodes }\end{array}$ & potentiometry & anions and cations & titrimetry, enzymes \\
\hline $\begin{array}{l}\text { Polarographic } \\
\text { electrodes }\end{array}$ & amperometry & $\begin{array}{l}\text { transition and } \\
\text { heavy metal cations; } \\
\text { as detectors in HPLC }\end{array}$ & - \\
\hline Clark electrode & amperometry & oxygen, halothane & in enzyme electrodes \\
\hline $\begin{array}{l}\text { Piezo-electric } \\
\text { crystal }\end{array}$ & $\begin{array}{l}\text { piezo-electric } \\
\text { mass detn. }\end{array}$ & $\begin{array}{l}\text { gases, volatile } \\
\text { liquids }\end{array}$ & - \\
\hline $\begin{array}{l}\text { Conductance } \\
\text { sensor }\end{array}$ & conductivity & - & $\begin{array}{l}\text { enzyme-catalysed } \\
\text { reactions }\end{array}$ \\
\hline Thermistors & $\begin{array}{l}\text { measurement of } \\
\text { reaction enthalpy }\end{array}$ & - & $\begin{array}{l}\text { exothermal bio- } \\
\text { chemical reactions }\end{array}$ \\
\hline
\end{tabular}

A schematic of the path of light inside a fibre is shown in Fig. 1. Light is totally reflected inside the fibre when the refractive index of the core is higher than that of the cladding.

In a typical fibre application for sensing purposes, light is focused into the core and guided to a sample to produce an analytical information there. The optical signal returns through the same (or another) fibre, and its intensity is measured. The input beam may be considered as the question, and the returning light as the encoded answer.

Optical waveguides can be useful in biosciences because they have minute diameters and high tensile strength. Therefore, they are of potential utility in the development of optical biosensors (3). This is a rapidly growing field of research, and the state of the art in chemical and biochemical sensors has been reviewed in several articles (411). This sensor type has certain advantages over other types (Table 1), and sensing with fibres can be superior to conventional spectroscopy because of a high degree of spatial flexibility, the ease of miniaturisation, and a well-defined localisation of optical measurement.

The first sensors designed to collect information via fibre-optics relied on the fact that alterations in a specific physical property of a medium being sensed would cause a predictable change in the light transmission characteristics of a fibre. In this case, the physical perturbation (such as a temperature change) interacts with the fibre to directly modulate the light intensity (3). These "sensors" are in fact simple optical fibres, with all their inherent advantages over conventional photometric methods. These sensors are referred to as intrinsic fibre sensors and usually lend themselves to measurement of physical parameters only.

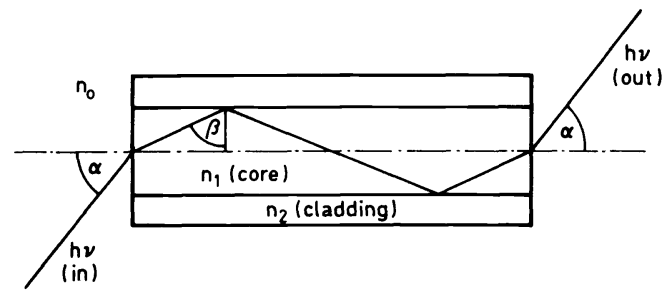

Fig. 1. Path of light inside an sptical waveguide

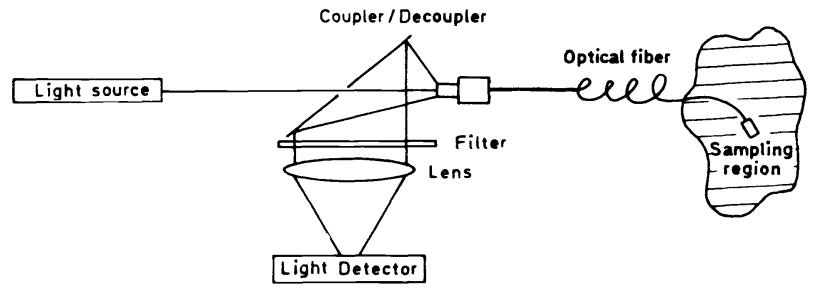

Fig. 2. Optical arrangement for performing fibre-optic analyses 
Extrinsic sensors, in contrast, measure an optical parameter not associated with the fibre itself. Two possibilities exist. In the first one, the intrinsic optical properties of a medium such as blood are monitored. A representative example is the determination of blood oxygen saturation by measuring its colour. These sensors are sometimes referred to as 1st generation extrinsic fibre sensors.

However, only a limited number of chemical species (namely, those having a colour or fluorescence by themselves) can be detected by first-generation (bare-ended) sensors described above. Moreover, these methods are of limited selectivity and selectivity in case of complex mixtures.

By combining the well-established indicator chemistry with fibre-optics and immobilisation techniques, a quite new technology has emerged in the past years that allows the construction of more selective and sensitive fibre-optic chemical sensors (FOCSs). They are capable of measuring analytes via chemical or physical processes occurring at some sort of indicator chemistry at the fibre end (2nd generation sensors). These sensors do not rely on the spectral properties of the sample and lend themselves to optical measurement of non-coloured and non-fluorescent analytes.

\section{ADVANTAGES OF FIBRE-OPTIC SENSORS}

Depending on the field of application, optical fibre sensing can offer advantages over other sensor types that make them particularly useful in biomedical sciences. The following are considered to be most important.

(a) Optical sensors do not require a reference signal such as is necessary in all potentiometric methods where the difference of two absolute potentials is measured.

(b) The ease of miniaturisation allows the development of very small, light, and flexible instrumentation. This is of great utility in case of minute sample volumes and in designing small catheters for invasive sensing in clinical chemistry and medicine.

(c) Because the primary signal is optical, it is not subject to electrical interferences by, e.g., static electricity of the body or surface potentials of the sensor head. Fibres do not present a risk to patients since there are no electrical connections to the body.

(d) Analyses can be performed in almost real-time and no sampling with its inherent drawbacks is necessary.

(e) Coupling of small sensors for different analytes to produce a bundle of small size allows simultaneous monitoring of various analytes such as $\mathrm{pH}$, oxygen and carbon dioxide by hybrid sensors without cross-sensing of the single strands.

\section{DISADVANTAGES OF FIBRE-OPTIC SENSORS}

Notwithstanding the significant advantages over other sensor types, fibre sensors exhibit the following disadvantages.

(a) Ambient light can interfere. Consequently, the sensor has to be applied in a dark environment or must be optically isolated, or its signal must be encoded to be able to differentiate it from background light.

(b) Sensors having immobilised indicator phases are likely to have limited longterm stability because of photobleaching or wash-out.

(c) Sensors with immobilized $\mathrm{pH}$ indicators or chelating reagents have limited dynamic ranges as compared to electrodes since the respective association equilibria obey the mass action law. The corresponding plots of optical signal versus log of analyte concentration are sigmoidal rather than linear as in the case of the Nernst relation.

(d) Commercial accessories of the optical system are not optimal yet. Stable and long-lived light sources, less expensive lasers, more intense blue LEDs, better connectors, terminals and optical fibres are needed. The lack of violet LEDs is particularly annoying.

\section{BIOMEDICAL FIELDS OF APPLICATION}

It is widely accepted now that the greatest field of fibre sensor application is sensing clinically and biochemically important analytes such as serum electrolytes, metabolites, enzymes, co-enzymes, immunoproteins, and inhibitors. Sensors responding to these parameters are frequently called biosensors $(5,6,10)$. They will be used in-vivo for continuous monitoring of the critically 111 and as devices for testing blood samples invitro. Continuous measurements of critical parameters which give warning of life-threatening trends such as pH, oxygen, carbon dioxide, and blood pressure are well established in principle. One may expect from fibre-optics to see continued improvements in biocompatibility, signal stability, ease of calibration, and sterilisation. A selection of potential biomedical sensor applications is compiled in Table 2. 
Table 2. Potential Biomedical Sensor Applications

$\begin{array}{lll}\text { Application } & \text { Mode } & \text { Example } \\ \text { Critical care } & \text { in vivo } & \mathrm{O}_{2}, \mathrm{CO}_{2}, \mathrm{pH}, \text { temperature, } \mathrm{K} \\ \text { Acute diagnosis } & \text { in vitro } & \begin{array}{l}\text { glucose, therapeutic drugs } \\ \text { glucose (artificial pancreas) }\end{array} \\ & \text { in vitro } & \begin{array}{l}\text { in } \\ \text { infood chemistry, abnormal function, }\end{array}\end{array}$

Apart from sensors for analytes which are present in relatively high concentrations, there is now a substantial demand for biosensors for substrates being present in considerably lower concentrations. It is assumed that in these cases fluorosensors will be of particular utility by virtue of the sensitivity of fluorimetry. Most likely, methods known for low-concentration analytes such as hormones, steroids, thyroid function constituents, pregnancy markers and therapeutic drugs will be adapted to continuous sensing. The interaction of antibodies with antigens, a process known to be of outstanding selectivity, can also be followed by optical methods. Evanescent wave sensors with immobilized antibodies on the waveguide surface, fluorescence polarization studies of labeled binding partners, fibre-optic time decay measurements, or combinations thereof, offer numerous sensing possibilities.

Before going into some details of spectrometry through fibres it appears useful to recall a few fundamental aspects of waveguides.

\section{OPTICAL WAVEGUIDES}

When light hits the interface between an optically thicker and optically thinner phase, it will suffer total reflection when the angle of incidence exceeds a certain critical angle $\underline{B}$ (Fig. 1). The relation between the two refraction indices and $\underline{B}$ is given by

$$
\sin B=\underline{n}_{2} / \underline{n}_{1}
$$

1.e., the ratio of the indices of refraction of core $\left(\underline{n}_{1}\right)$ and cladding $\left(\underline{n}_{2}\right)$ of the fibre. Fig. 1 shows that the distal end of a waveguide can only accept and release light within a certain angle $\underline{\alpha}$ which is governed by the two refraction indices again:

$$
\underline{n}_{0} \cdot \sin \alpha=\left(\underline{n}_{1}^{2}-\underline{n}_{2}^{2}\right)^{1 / 2}
$$

with $\underline{n}_{0}$ being the index of refraction of the medium outside the fibre, usually air or water. We see that the index of refraction of core and cladding determine two of the most important optical parameters of a waveguide. $\underline{n}_{0} \cdot \sin \alpha$ is the so-called numerical aperture.

When entrapped in a fibre or a similar waveguide, light can propagate over large distances, will be released at the fibre end and can hit a sample there. But although total reflection implies no light attenuation, small losses occur due to absorption in the medium, discontinuities in the reflecting surface, and reflections at the surfaces when light enters and leaves the medium. This limits the distance over which spectroscopy in the visible can be performed, to several hundred meters.

One of the decisive advantages of "optical wires" over electric wires is the rate of information flux. An optical fibre can transmit much more information simultaneously, for instance blue and yellow light in one direction, and green and red light in the other. The advantage for the analytical spectroscopist lies in the possibility to use one fibre for multi-wavelength spectroscopy, for instance by guiding the excitation beam from the source to the sample, and, simultaneously, fluorescence or Raman scatter to a detector.

Apart from measuring the intensity of reflected light or fluorescence produced at the distal end of a fibre, another possibility exists for performing optical spectroscopy with waveguides. It rnsults from the fact that light, when totally reflected at a dielectric interface, penetrates to some extent into the optically thinner phase. This phenomenon is referred to is evanescent wave (12) and is schematically shown in Fig. 3. The depth of penetration into the rare phase ( $d$ in Fig. 3) is determined by the wavelength of light and the refraction indices of core and cladding. Typically, $d p$ ranges from 50 to $1200 \mathrm{~nm}$ for visible light and usual glass or plastic fibres. Within 


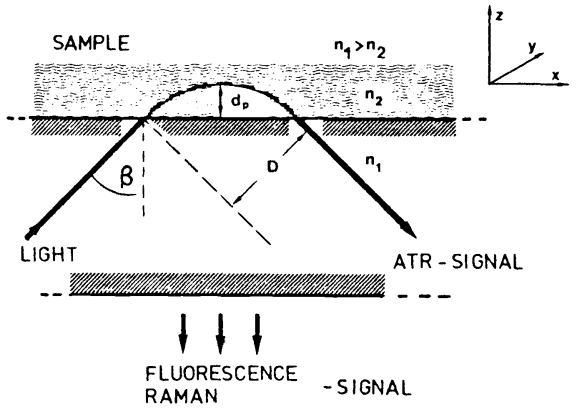

Fig. 3. Schematic of the total reflection of light occurring at a di-electric interface and showing its evanescence into the optically thinner phase.

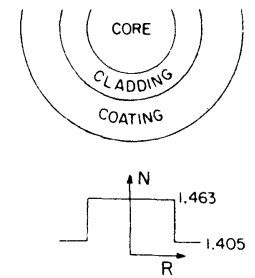

(a)

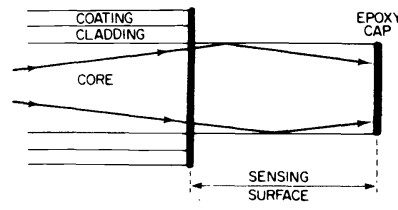

(b)
Fig. 4. (a) Cross-section through a fibre and demonstration of step-index profile; (b) schematic of a fibre sensor based on evanescent wave spectrometry.

this depth, the evanescent wave can be absorbed by a sample solution outside the core. It may induce its fluorescence or Raman scatter. Evanescent wave spectroscopy is obviously of potential utility to probe thin layers, for example of proteins or antibody-antigen interactions at surfaces, or monodispersed microspheres (13).

Fig. 4 shows a cross-section of a fibre, the differences in refraction indices of core and clad, and a typical sensor application. The sensing surface can be, for instance, an immobilised $\mathrm{pH}$ indicator. A reflecting cap is attached to the distal end in order to reflect the signal. Measurement of absorption, fluorescence, and Raman scatter is possible.

\section{REPRESENTATIVE FIBRE-OPTIC SENSORS}

In view of the number of optical biosensors that has been reported in the past years it is certainly impossible to present all of them. Rather, a few typical representatives shall be discussed in some detail in order to provide an idea on the possibilities of the technique.

\section{Physical sensors}

Among the sensors that have been proposed for various physical parameters, the fibreoptic temperature sensor developed by the ASEA Corp. 14) s a most elegant one and shall be presented here as a typical example. Its operation principle is schematically shown in Fig. 5 .

A red LED acting as a light source excites a small gallium arsenide crystal attached to the end of a 100- $\mu \mathrm{m}$ fibre. The crystal has a strongly temperature-dependent nearinfrared fluorescence that returns through the same fibre along with some scattered red

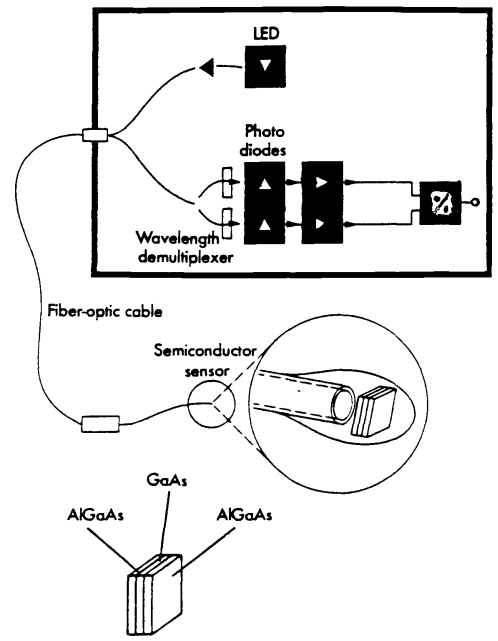

Fig. 5. Optical arrangement of the ASEA fibre-optic thermometer and exploded view of the sensor tip, consisting of a GaAs semiconductor material displaying a highly temperature-dependent luminescence. 


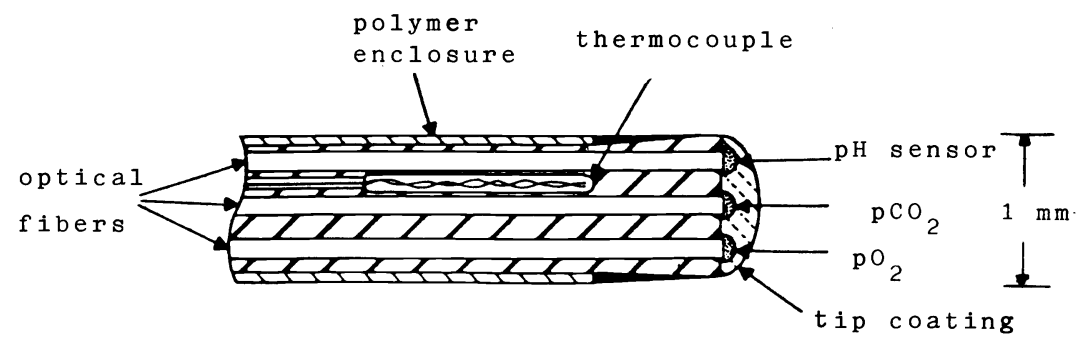

Fig. 6. Cross-section of a 1-mm fibre-optic catheter for continuous monitoring of oxygen, carbon dioxide, and pH.(From ref. 16).

light. After passing a beam splitter, light is guided through two filters. The one blocks reflected red light and transmits IR fluorescence; the other blocks fluorescence and transmits red light. The two light intensities are measured and ratioed to give a signal that is related to temperature. A $+/-0.1^{\circ} \mathrm{C}$ resolution is achieved in the 0 to $200^{\circ} \mathrm{C}$ temperature range and over a maximal distance of 500 metres.

\section{Chemical sensors}

Oximetry. The differences in the absorption spectra of oxyhaemoglobin and haemoglobin can be exploited to measure oxygen saturation of blood. Because of its high absorbance at analytical wavelengths below $600 \mathrm{~nm}$, practically all work has been performed at wavelengths close to or beyond the red end of the visible spectrum. The advent of red and infrared LEDs along with red-sensitive photodiodes has considerably contributed to stimulate these experiments.

Blood reflectance measurement are generally performed at $660 \mathrm{~nm}$. Internal standardisation is achieved by measuring reflection at around $805 \mathrm{~nm}$ which is one of the isosbestic wavelengths in the absorption spectra of haemoglobin and oxyhaemoglobin. Only recently, an implantable sensor was developed that can measure both blood oxygensaturation and haematocrit from reflectance data (15).

Blood Gas and $\mathrm{pH}$ Sensors. The most advanced technology known so far is packed into a small triple sensor only $1 \mathrm{~mm}$ in diameter (16). It measures $\mathrm{pH}$, oxygen and co on-line and can be inserted into blood vessels during operations. Fig. 6 shows the tip of this triple sensor, consisting of three 100 micrometer thick fibres with three different analyte-sensitive layers at their end, along with a conventional thermocouple for measuring temperature. All three parameters are determined by fluorimetry: Oxygen via its quenching effect upon an aromatic hydrocarbon, $\mathrm{pH}$ via the changes in fluorescence of a covalently immobilised indicator, and carbon dioxide via optically detected changes in the $\mathrm{pH}$ of a buffer solution entrapped in a polymer.

A first version of this sensor ("Gas-Stat") that can monitor blood gases and pH in an extracorporeal loop is commercially available since 1984 (17). Arterial and venous oxygen and carbon dioxide $\mathrm{pH}$, and temperature can continuously be determined during cardiopulmonary bypass surgery. The system consists of a microprocessor-based instrument, bifurcated fibre-optic cables, and a disposable sensor head with fluorescent spots sensitive to the respective analytes.

Blood Electrolytes. Only a few approaches have been made to sense blood electrolytes $(18,19)$, probably because of a lack of suitable indicators for alkali and earth alkali metals that respond at near-neutral pH's. A quite new sensing principle for these ions is based on fluorescent dyes sensitive to a potential formed at a lipid-water interface (20). The method is similar to the one applied in ion-selective electrodes, except that the potentiol is measured optically. Fig. 7 shows how a typically ion-selective optode

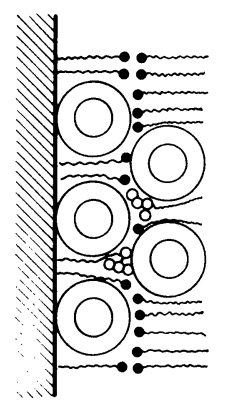

Fig. 7. Schematic of a sensing layer in an ionselective optode (ISO). L, lipid layer (arachidic acid); RhoB, lipidsoluble rhodamine dye; Val, valinomycin; G, glass support; $S$, sample solution. 


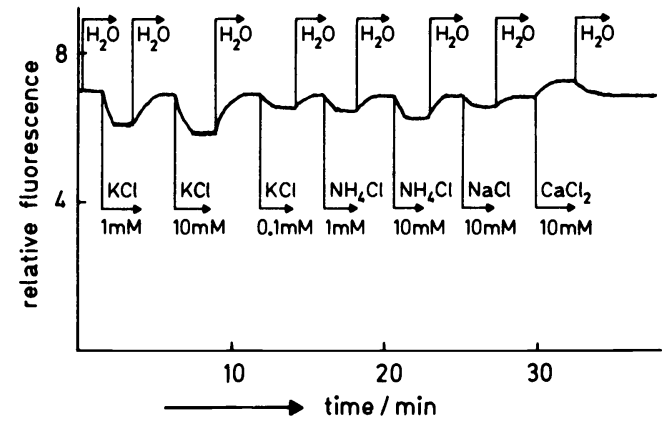

Fig. 8. Typical fluorescence response of an ISO towards potassium at various concentrations.

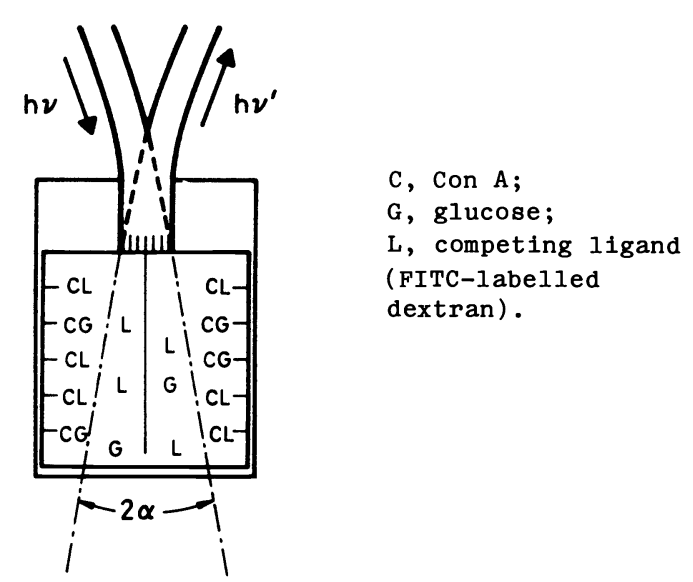

Fig. 9. Glucose sensor based on competitive binding.

(IOS) is blind up. In essence, it consists of a solid support, covered with a LongmuirBlodgett-type lipid bilayer and stained with a potential-sensitive indicator. The selectivity towards different metal ions is provided by addition of suitable ion carriers such as valinomycin.

The dye responds to the potential that is created at the interface when potassium is complexed into the lipid phase. The Langmuir-Blodgett technique is particularly useful because it allows the fabrication of very thin and well-defined layers where the potential created per $\mathrm{cm}$ is almost maximum.

The advantage of such a system is that it can be constructed similarly for a number of electrolytes, simply by changing the ion carrier. Fig. 8 shows the response of a typical sensor towards potassium ion. The selectivity over sodium ion is between 1000 and 100,000 .

Glucose. The first optosensor for glucose was described in 1980 and is based on the measurement of oxygen consumed as a result of the enzymatic oxidation of glucose by glucose oxidase in the presence of catalase (5, and refs. cited there). An oxygen optode (based on fluorescence quenching) acts as a transducer for the enzymatic reaction. Glucose sensors exploiting the decrease in $\mathrm{pH}$ as a result of glucose oxidation to glucuronic acid have not been reported so far, nor ones that are based on measurement of hydrogen peroxide that is formed during the same reaction.

A glucose sensor exploiting the phenomenon of competitive binding of substrates to an enzyme has been described by Schultz (21). Fluorescein-labelled dextran competes with glucose for binding to concanavalin A (Con A) immobilised on sepharose inside a minute reaction chamber. The fibre optic "sees" only the free ligands within an angle $2 \alpha$. Glucose can freely diffuse into and out the fibre, but dextran-immobilised fluorescein can not. The more glucose is present, the more dextran will be released by glucose and will diffuse into the cone (of angle $2 \alpha$ ) vined by the fibre. Fig. 9 shows a schematic of the device.

This sensor principle is of particular interest because it has a broad potential for application. It may be adapted to any analytical problem for which a specific competitive binding system can be devised.

Proteins, Antigens, and Antibodies. Detection of antibody-antigen reactions has been demonstrated by using the evanescent wave sensing technique. The general characteristics of these immunoassay systems includes kinetic monitoring of the immunological reactions without major interferences from the bulk solution and without aformal separation step of antibody-bound from free antigen.

A useful example is provided by the determination of IgG by a sandwich immunofluoroassay (10). First, immobilised sheep antiserum is incubated with antigen to form one half of the sandwich. After washing out excess IgG the solid phase complex was reacted with FITC-labeled antiserum and the reaction monitored for $15 \mathrm{~min}$. Fig. 10 shows the detection of the binding process on the surface of a slide. 


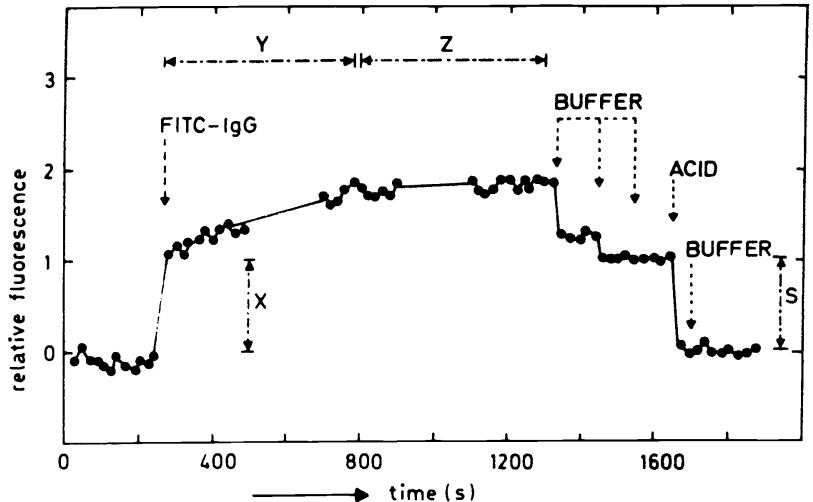

Fig. 10. Time course of the fluorescence measured by the evanescent wave technique in a thin quartz slide, when FITC-labelled anti-IgG is added to glass-adsorbed IgG.

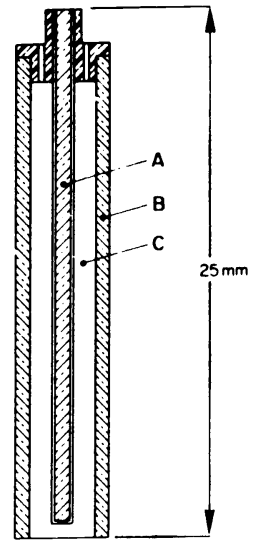

A, fibre covered with antibody plus labelled antigen;

B, capillary tubing;

C, Cerined sample volume.

Fig. 11. Disposable fibre sensor for determination of antigens or antibodies via competitive binding.

Following the injection of FITC-labeled anti-IgG, a rapid increase in fluorescence intensity is observed $(X)$. This is mainly due to free molecules fluorescing within the penetration depth of the evanescent wave. Secondly, a slower increase in binding over the next $500 \mathrm{~s}$ is evident, reaching a plateau after approximately $600 \mathrm{~s}$. The curve obtained when no antigen was present shows fluorescence because of the presence of free molecules, but lacks the rapid binding event.

A simple principle for performing fibre-optic immunoassays has been proposed by Hirschfeld (22). The disposable sensor consists of a capillary tubing having an axially disposed optical fibre (Fig. 11). The dimensions are chose so to allow the tube to fill itself by capillary action when immersed into the sample. The fibre is covered with a monolayer of antibody and a controlled amount of labelled antigen. When the blood sample enters the reaction chamber, some antigen in the blood will displace labelled antigen from the fibre surface, resulting in a decrease in fluorescence which is "seen" by the evanescent wave. The decrease can be related to the antigen concentration.

A fibre-optic sensor for the direct kinetic determination of enzyme activities (24) is based on the immobilisation of chromogenic or fluorogenic substrates at the end of a fibre. When hydrolysed by an enzyme, the substrate is converted into a coloured and highly fluorescent product. The increase in fluorescence with time can be correlated to the activity of the enzyme (an esterase). It should be kept in mind that this type of probe is not a sensor, since it acts irreversibly. To differentiate it from a true sensor, I suggest to use the term probe exclusively for this type of device, and to confine the word "sensor" to devices that act continuously and reversibly.

The principle can be extended to other hydrolases as well. The synthetic substrates do not present a health risk when applied in vivo since it is the non-toxic acid or sugar component that is released, whereas the dye remains immobilised. The probe can be used several times, for instance first in a calibration step, and then for sample sensing. The sensitive layer at the fibre end is preferably a disposable thin layer of immobilised substrate. Fig. 12 shows how the signal develops with time and the rate of non-enzymatic hydrolyis for comparison.

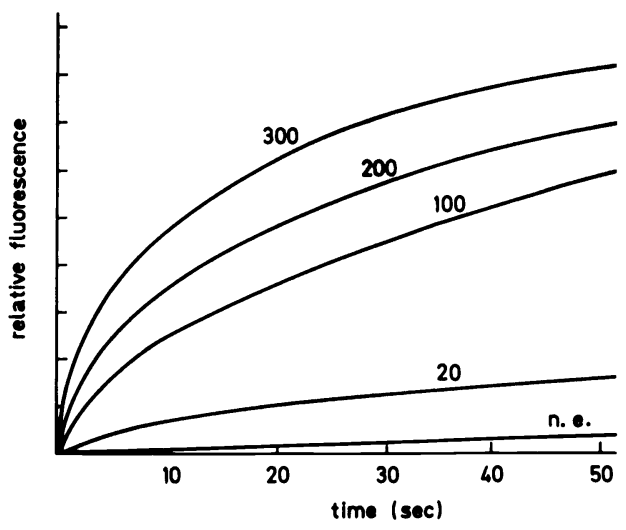

Fig. 12. Response of a fibre probe for the enzyme carboxylesterase. The figures refer to micrograms of enzyme per ml solution, into which the probe was immersed, and n.e. is the signal due to non-enzymatic hydrolysis. 


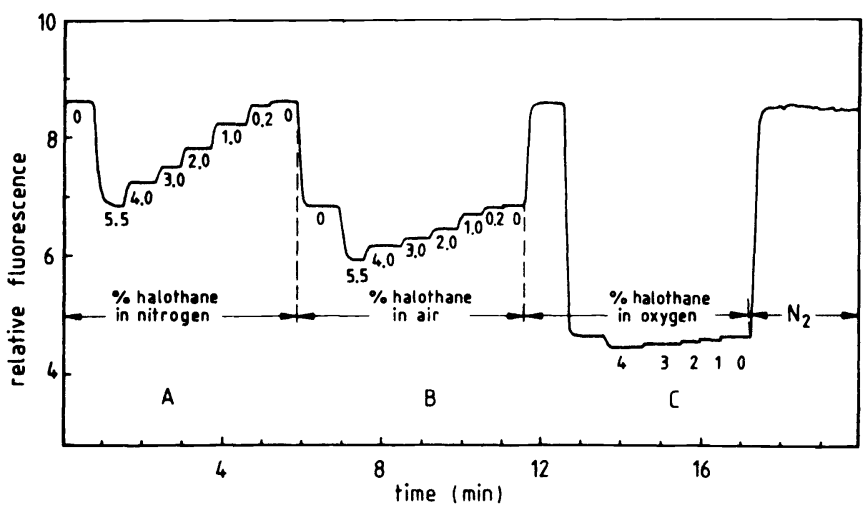

Fig. 13. Optical signal change obtained with an fluorosensor for determination of oxygen and/ or halothane.

Drugs and Anaesthetics. A fibre-optic sensor for the widely used inhalation narcotic halothane in the presence of varying levels of oxygen was presented (24). It is based on dynamic fluorescence quenching of a suitable fluorophor by halothane in a silicone rubber matrix. Interferences by molecular oxygen are taken into account by a second sensor layer covered with PTFE. The latter is halothane-impermeable so that the concentration of halothane can be calculated from the difference in the two signals.

The response of a sensor membrane towards halothane admixed to nitrogen, air, and oxygen is shown in Fig. 13. The signal changes are large enough to allow a quantitation of halothane, or oxygen, or both with a precision of $+/-0.1 \%$ for halothane, and $+/-1$ Torr for oxygen in the physiological concentration range. The sensor is practically specific for the two analytes since other anaesthetics have little or no effect; $0.2 \%$ fialcihane is easily detectable in air.

\section{FINAL REMARKS}

The design of new fibre sensors is a quite active area of research with a number of direction that will be followed in the future. Aside from conventional intensity measurements, other parameters will be used. We are cus rently developing an oxygen sensor based on the decay time of the indicator as an informaition carrier. The advantage of such a system is that it has an internal reference (viz., the excitation pulse). Further features include a long lifetime, no bleaching and wash-out effects, no drifts due to lamp and detector sensitivity fluctuations, long calibration intervals, and linear calibration plots (25). The measurement of the time lag between excitation and emission is in fact some sort of internal referencing.

Sensors based on energy-transfer sensors are another current trend that will provide wu." possibilities and a greater flexibility in the choice of dyes (26) and with particular advantages for studying immunological binding reactions, since the efficiency of dipoledipole energy transfer decreases with the 6 th power $n+$ distance. Energy-trarisfer-based sensors can be devised for both ground-state and excited-state processes, and both trivial (re-absorption) and dipole-dipole mechanisms offer certain advantages.

Oxidases, a large group of enzymes producing hydrogen peroxide, can be assayed now with a new optode for hydrogen peroxide presented at this symposium (27), and a number of chemiluminescence-associated reactions awaits to be exploited in fibre-sensor technology .

It is hoped that this article can convey some of the enthusiasm that is currently shared by researchers in this field. It is felt that fibre-optic sensors can significantly contribute to the development of new sensing techniques and that, for entrepreneurs, the opportunity exists to participate in a rapidly growing segment of the market for clinical analyses, although there may be tough competition from other sensor instrumentation such as electrodes, FETs, piezo-electric devices, Fourier-transform IR spectroscopy, calorimetry, and dry-reagent chemistry. Most probably, an interdisciplinary approach to the solution of existing hurdles will be essential.

\section{REFERENCES}

(1) I. Chabay, Optical Waveguides, Anal. Chem. 54, 1071A (1982).

(2) J. C. Dale, Fiber Optics, CRC Press, Boca Raton, FL, 1984.

(3) T. Giallorenzi et al., Optical Fiber Sensor Technology, IEEE J. Quant. Electr. QE18, 626 (1982). 
(4) T. Hirschfeld, T. Deaton, F. Milanovich, and S. Klainer, Feasibility of Monitoring Groundwater Contaminants, Opt. Eng. 22, 527 (1983).

(5) D. W. Lubbers and N. Opitz, Optical Fluorescence Sensors for Continuous Measurement of Chemical Concentrations in Biological Systems, Sensors \& Actuators 4 , 641 (1984).

(6) J. I. Peterson and G. G. Vurek, Fiber-optic Sensors for Biomedical Applications, Science224 (1984) 123 .

(7) T. Hirschfeld, J. B. Callis and R. B. Kowalski, Chemical Sensing in Process Analysis, Science 226, 312 (1984).

(8) 0. S. Wolfbeis, Fluorescence Optical Sensors in Analytical Chemistry, Trends Anal. Chem. 4,184 (1985).

(9) W. R. Seitz, Chemical Sensors Based on Fiber Optics, Anal. Chem. 56, 16A (1985).

(10) J. F. Place, R. M. Sutherland, C. Dăhne, Opto-electronic Immunosensors: A Review of Optical Immunoassay at Continuous Surfaces, Biosensors 1, 321 (1985).

(11) 0. S. Wolfbeis, Analytical Chemistry with Optical Sensors, Z. Anal. Chem. 325,387 (1986).

(12) G. J. Muller, Spectroscopy with the Evanescent wave in the Visible Region of the Spectrum, Am. Chem. Soc. Symp. Ser. 102: Multichannel Image Detectors (J. Talmi, ed), p. 239 (1979).

(13) J. D. Andrade et al., Remote Fibre Optic Biosensors Based on Evanescent Excited Fluorolmmunoassay: Concepts and Progress, IEEE Trans. Electr. Dev. ED-32, 1175 (1985).

(14) ASEA Innovation, S-72183 Västeras, Sweden.

(15) J. M. Schmitt, J. D. Meindl, F. G. Mihm, An Integrated Circuit-Based Optical Sensor for In-vivo Measurement of Blood Oxygenation, IEEE Trans. Biomed. Eng. BME-33, 98 (1986).

(16) Gehrich et al., Optical Fluorescence and Its Application to an Intravascular Blood Gas Monitoring System, IEEE Trans. Biomed. Eng. BME-33, 117 (1986).

(17) Cardiovascular Devices Inc., 2801 Barranca Rd., Irvine, CA 92714, USA.

(18) E. Urbano, H. Offenbacher and 0. S. Wolfbeis, Optical Sensor for Continuous Determination of Halides, Anal. Chem. 56, 427 (1984).

(19) Z. Zhujun, J. L. Mullin and W. R. Seltz, Optical Sensor for Sodium Based on IonPair Extraction and Fluorescence, Anal. Chim. Acta 184, 251 (1986).

(20) 0. S. Wolfbeis, B. P. H. Schaffar, An Ion-selective Optode for Potassium, Anal. Chim. Acta, in press.

(21) J. S. Schultz, Optical Sensor for Plasma Constituents, US-Pat. 4.344,438 (1982).

(22) T. Hirschfeld, Fluorescent Immunoassay Employing Optical Fiber in Capillary Tube, US-Pat. $4.447,546$ (1984).

(23) 0. S. Wolfbeis, Fiber-Optic Probe for Kinetic Determination of Enzyme Activities, Anal. Chem. 58, 2874 (1986); Ger. Pat. 3.701.833.

(24) O. S. Wolfbeis, H. E. Posch and H. K. Kroneis, Fiber Optical Fluorosensor for Determination of Halothane and/or Oxygen, Anal. Chem. 57, 2556 (1985); US-Pat. $4.580,059$

(25) M. E. Lippitsch, J. Pusterhofer, M. J. P. Leiner, O. S. Wolfbeis, Fibre-optic Oxygen Sensor with the Decay Time as the Information Carrier, Abstracts of papers of the II. Intl. Symp. Quant. Luminesc. Biomed. Sci., Ghent (Belgium), May 11-14, 1987.

(26) D. M. Jordan, D. R. Walt, F. P. Milanovich, Physiological pH Fiber Optic Chemical Sensor Based on Energy Transfer, Anal. Chem. 59, 437 (1987).

(27) 0. S. Wolfbeis and H. E. Posch, Optical Sensor for Hydrogen Peroxide, Abstracts of papers of the II. Int1. Symp. Quant. Luminesc. Biomed. Sci., Ghent (Belgium), May 11-14, 1987. 